\title{
Calidad de vida de persona con heridas complejas en Cartagena, Colombia
}

\section{The quality of life for people suffering from complex wounds in Cartagena, Colombia}

\author{
Estela Melguizo-Herrera, Adriano A. Díaz-Gómez y Martha Osorio-Lambis \\ Facultad de enfermería, Universidad de Cartagena. Cartagena, Colombia. esmelguizo@hotmail. \\ com,agaton1964@yahoo.es, heridascomplejas@hotmail.com
}

Recibido 17 Enero 2011/Enviado para Modificación 5 Julio 2011/Aceptado 8 Octubre 2011

\section{RESUMEN}

Objetivo Estimar la Calidad de Vida Relacionada con Salud-CVRS- de personas con heridas complejas que reciben cuidados en una Institución Prestadora de Servicios de Salud Extramural en Cartagena, durante mayo-junio de 2010.

Materiales y métodos Estudio cuantitativo, descriptivo, cuya población y muestra fueron 93 personas adultas que reciben cuidados en el programa de heridas complejas de una Institución Prestadora de Servicios de Salud Extramural de la ciudad de Cartagena. Se aplicó un cuestionario sobre características socio demográfico, una guía de observación sobre las características de las heridas y el "Índice Multicultural de Calidad de Vida". Para el análisis estadístico se empleó el software SPSS 12.

Resultados El promedio de edad fue de 59,3 años; predominó género femenino, 61,3 $\%$; estado civil casado, 43,0 \%; ocupación hogar, 38,7 \%; escolaridad primaria, 38,7 $\%$; y estratos socio-económicos 2 y $3,68,8 \%$. El 93,5\% de los participantes tienen afiliación a la seguridad social en salud. Las heridas complejas se caracterizaron por presentar mayor porcentaje en la fase proliferativa de la cicatrización, 80,6 \%; medir de seis a diez centímetros, 63,4 \%; no reportar infecciones, $85 \%$; no presentar amputación, 90,3 \%; ni dolor relacionado con la herida, 52,7 \% y presentar limitación física relacionada con la herida, $66,7 \%$.

Conclusiones Las personas perciben su CVRS como mala, resaltando con baja puntuación las dimensiones de plenitud personal, funcionamiento ocupacional y bienestar físico y psicológico.

Palabras Clave: Calidad de vida, salud, promoción de la salud, atención domiciliaria de salud (fuente: DeCS, BIREME).

\section{ABSTRACT}

Objective Estimating the health-related quality of life (HRQOL) of people suffering complex wounds who were receiving care in an extramural health service provider institution in Cartagena during May-June 2010. 
Materials and methods This was a quantitative, descriptive study where the population/sample consisted of 93 adults receiving care in an extramural health service provider institution's complex wounds program in the city of Cartagena. A questionnaire regarding socio-demographic characteristics, an observational guide on the characteristics of injuries and the Multicultural Quality of Life Index were used. SPSS 12.0 software was used for the statistical analysis.

Results Mean age was 59.3 years, females predominated (61.3\%), $43.0 \%$ were married, $38.7 \%$ were housewives, $38.7 \%$ had finished primary education, $68.8 \%$ came from socioeconomic levels 2 and 3 and $93.5 \%$ of the participants had health social security affiliation. Complex wounds were characterised by a greater percentage being in the proliferative phase of healing $(80.6 \%)$, measuring six to ten inches $(63.4$ $\%)$ no infection being reported (85\%), no amputation (90.3\%) or wound-related pain $(52.7 \%)$ and presenting physical limitations related to the wound $(66.7 \%)$.

Conclusions People perceived their HRQOL as poor, their low scores highlighting low personal fulfillment, occupational functioning and physical and psychological wellbeing.

Key Words: Quality of life, public health, wound, injury (source: MeSH, NLM).

$\mathrm{P}$ or calidad de vida relacionada con salud (CVRS), se entiende "la percepción subjetiva, influenciada por el estado de salud actual, de la capacidad para realizar aquellas actividades importantes para el individuo" (1).

La CVRS puede estar influida por la presencia de heridas, la cuales pueden estar acompañadas de cambios en la vida diaria, sufrimiento, dolor, limitaciones, dependencia, alteración de la autoestima, temor, angustia y aislamiento, entre otros (2). Investigaciones han reportado asociación entre CVRS y cicatrización (3-5); inflamación y tamaño de la herida (6); dolor (7); impedimentos físicos consecuencias de las heridas (8) y amputación mayor o menor (4).

En Colombia el cuidado ambulatorio de las heridas complejas, es remitido a la Empresa Promotora de Servicios de Salud (EPS) a la que está afiliado el paciente, y queda a cargo de un familiar o personal de enfermería. Cuando el cuidado queda a cargo del familiar, este es "entrenado" por enfermeras, quienes semanalmente suministran los insumos necesarios y supervisan la evolución de la herida.

Dado que el Programa de Heridas Complejas de la Empresa Unipersonal de Servicios Integrales de Medicina y Enfermería (SIME E.U.), en 
Cartagena, es el único a cargo de enfermeras profesionales, se decidió llevar a cabo este estudio.

SIME E.U., es una Institución Prestadora de Servicios de Salud (IPS), extramural, autorizada por el Departamento Administrativo Distrital de Salud, del Distrito de Cartagena; bajo el número 130001745 01, conformada por profesionales y personal de apoyo, capacitados para ofrecer servicios de salud a la población de Cartagena y de Bolívar.

El Programa Clínica de Heridas Complejas de la empresa SIME E.U, se define como el conjunto de procedimientos y cuidados encaminados al restablecimiento pleno de la salud de los pacientes que presentan lesiones de la piel, utilizando los conocimientos y los elementos biotecnológicos y terapéuticos más avanzados, realizados por profesionales de enfermería. Teniendo en cuenta lo anterior, los pacientes incluidos en el programa no requieren curaciones diarias sino ínter diarias, cada quinto o séptimo día, de acuerdo a la evaluación inicial y características de las heridas. Las heridas más frecuentemente atendidas son ulceras vasculares, pie diabético, ulceras por presión, heridas quirúrgicas abiertas, heridas tumorales entre otras.

La falta de información sobre la CVRS de este grupo de personas, representa una dificultad para el Sistema de Seguridad Social en relación con la creación e implementación de programas que disminuyan la exclusión social y mejoren la CVRS de esta población. No se han encontrado estudios sobre la CVRS de personas con heridas complejas en Cartagena.

El objetivo de este estudio fue estimar la CVRS de personas adultas, con heridas complejas que reciben cuidados en una Institución Prestadora de Servicios de Salud Extramural en Cartagena, durante junio-julio de 2010.

En la literatura revisada, el concepto de CVRS se observa dinámico, cambiante, multidimensional, sin consenso entre los autores, asociado al bienestar físico, mental y social y aparece como sinónimo de salud percibida (9) o estado de salud (1).

La CVRS, también es definida como: "el valor atribuido a la vida, ponderado por el deterioro funcional, las percepciones y condiciones 
sociales que son inducidas por la enfermedad, complicaciones, tratamientos y la organización política y económica del sistema asistencial" (10). Esta definición coincide en muchos aspectos con la propuesta de Botero (11) quien propone que CVRS es: "la capacidad que tiene el individuo para realizar aquellas actividades importantes relativas al componente funcional, afectivo y social, los cuales están influenciados por la percepción subjetiva".

Un análisis de la literatura disponible permite concluir que CVRS "surge como un concepto que hace referencia a valoraciones de la percepción de la salud por parte del individuo, acumulando tanto aspectos objetivos como subjetivos" (12).

El concepto de CVRS, en enfermería, según Bredow y Peterson, frecuentemente está asociado a enfermedades específicas y consideran, estos autores, que los componentes básicos de la calidad de vida tienen que ver con aspectos físicos, psicológicos, socioeconómicos y espirituales; afectados por el contexto y las características de los individuos (13).

Desde la psiquiatría, Mezzich et al, luego de revisar 21 propuestas de medición de calidad de vida, desarrollaron el "Índice Multicultural de Calidad de Vida" (QLI-Sp). La escala considera múltiples dimensiones de la CVRS, reflejando la complejidad del concepto $(14,15)$.

Con base en la revisión de literatura y la experiencia clínica, los investigadores definieron heridas complejas como aquellas extensas y/o profundas, que pueden presentar sangrado, con compromiso de tejidos subdérmicos, en las que puede o no existir perforación visceral y que tienden a presentar complicaciones inherentes al estado clínico de las personas, con evolución tórpida hacia el estancamiento o la cronicidad.

Ribu (6), encontró que la presencia de signos bioquímicos de inflamación (proteína $\mathrm{C}$ reactiva mayor de $10 \mathrm{mg} / \mathrm{L}$ ), índice brazo-tobillo menor de 0,9 ; tamaño de la ulcera mayor o igual de $5 \mathrm{~cm}$; nefropatía e índice de masa corporal menor de $25 \mathrm{Kg} / \mathrm{m}^{2}$, fueron variables que explicaron la varianza de la CVRS. La asociación más fuerte se produjo entre los signos bioquímicos de inflamación, tamaño de la úlcera y el funcionamiento físico.

En estudios realizados en Noruega, Norte América y Suecia, se encontró que la cicatrización por primera intensión es una variable asociada a la 
CVRS (3-5). A este respecto, Ribu L. (5), agrega que el funcionamiento social y la salud mental, fueron las dimensiones más afectadas por la no sanación de las úlceras.

La presencia de dolor la mayor parte del tiempo o todo el tiempo, mostró asociación clínica y estadísticamente significativa con el autoreporte de CVRS en personas con ulcera en pie diabético (7).

La presencia actual o el haber sufrido ulceras en miembros inferiores, en personas diabéticas, mostró gran impacto en la calidad de vida, en cuanto a la función física, funcionamiento físico y la movilidad (8). Así mismo, la amputación mayor o menor en las extremidades inferiores, es reportada como una variable asociada a bajos puntajes de CVRS (4). No se encontraron informes de investigación que abordaran la CVRS en personas con heridas complejas en Colombia.

En resumen, según las investigaciones, la CVRS es influenciada por aspectos como las fases del proceso de cicatrización, tamaño de la herida, presencia de dolor, amputaciones y limitación física relacionada con la herida.

\section{METODOLOGÍA}

Estudio descriptivo. La población y muestra estuvo constituida por todas las personas adultas del programa de Heridas Complejas de SIME E.U. (93 personas), durante los meses de junio-julio de 2010.

La información fue recolectada por dos enfermeras profesionales previamente capacitadas. Se aplicó un cuestionario para recolectar datos sobre las características socio-demográficas, una guía de observación elaborada por el grupo investigador para valorar las características de las heridas complejas que de acuerdo con la literatura encontrada están asociadas con la CVRS (3-8). Se diligenció el "Índice Multicultural de Calidad de Vida".

El "Índice Multicultural de Calidad de Vida" ha sido validado y aplicado en Perú $(16,17)$, Argentina (18), España (19), Cuba (20) y Estados Unidos (14). Es una escala confiable, que posee una alta consistencia interna, capaz 
de discriminar grupos en los que se presumen diferentes niveles de CVRS, eficiente y de fácil aplicación. Valores de ocho y más corresponden a excelente CVRS, mientras que valores menores de ocho, correspondieron a mala CVRS. Para el análisis estadístico se empleó el software SPSS $12.0^{\mathrm{R}}$.

Se considera que esta fue una investigación sin riesgo, donde la identidad de los participantes se mantuvo en el anonimato y se diligenció el consentimiento informado (21-23).

\section{RESULTADOS}

Participaron 93 personas, con un promedio de edad de 59,3 años, 61,3\% mujeres; 43,0 \% casados; 38,7 \% tenían nivel de escolaridad primaria; 38,7 $\%$ ocupación hogar, seguidos de 25,8 \% con ocupación independiente; 55,9 $\%$ manifestaron no disponer de recursos económicos para el cuidado de la herida; $38,7 \%$ viven en estrato $2 ; 75,3 \%$ disponen de cuidadores y $93,5 \%$ cuentan con afiliación a una EPS.

De acuerdo a la fase de cicatrización de las heridas, se observó que el $80,6 \%$ de las heridas se encontraban en fase proliferativa; $14,0 \%$ estaban en fase de maduración y $5,4 \%$ se encontraban en fase inflamatoria. El $63,4 \%$ de las heridas medían de seis a diez centímetros; $85,0 \%$ no reportó infecciones; $90,3 \%$ no presentaron amputación; $52,7 \%$ no manifestaron dolor relacionado con la herida y $66,7 \%$ presentaron limitación física relacionada con la herida.

La Tabla 1 ilustra sobre los promedios obtenidos de cada de las dimensiones de la CVRS y el promedio general de todas las dimensiones.

Tabla 1. Promedios de las dimensiones de la calidad de vida relacionada con la salud de las personas con heridas complejas Cartagena junio-julio

\begin{tabular}{lc}
\hline Dimensiones CVRS & Promedio \\
\hline Bienestar físico & 6,2 \\
Bienestar Psicológico/Emocional & 6,3 \\
Auto-Cuidado y funcionamiento independiente & 6,6 \\
Funcionamiento ocupacional & 5,5 \\
Funcionamiento interpersonal & 7,7 \\
Apoyo social-emocional & 8,4 \\
Apoyo comunitario y de servicio & 6,9 \\
Plenitud personal & 5,4 \\
Plenitud Espiritual & 9,3 \\
Percepción Global de calidad de vida & 6,9 \\
Promedio total de las dimensiones de la CVRS & 6,9 \\
\hline
\end{tabular}




\section{DISCUSIÓN}

Las personas con heridas complejas perciben tener una mala CVRS, resaltándose las bajas puntuaciones en las dimensiones plenitud personal, funcionamiento ocupacional y bienestar físico.

El predominio del sexo femenino difiere de los hallazgos de Da Silva (24), quien reporta que la mayoría de los participantes de su estudio fueron del sexo masculino $(53,3 \%)$.

El predominio de casados coincide con lo reportado por Da Silva (24), y difiere de los hallazgos de Ribu . (5). Igualmente Najman (25), considera que el principal determinante de un alto grado de calidad de vida parece ser la convivencia con la familia y la compañía a nivel social.

Los bajos niveles de escolaridad reportados coinciden con los informados por Ribu (5), y por Da Silva (24). El bajo nivel de escolaridad podría estar favoreciendo comportamientos de riesgo que generan heridas, complicaciones o limitaciones para buscar atención oportuna.

El alto porcentaje de personas con trabajos no formales o en actividades del hogar concuerdan con los hallazgos de Ribu (5), y de Da Silva (24). No se debe olvidar que los trabajos no formales y del hogar carecen de la seguridad ocupacional apropiada y cuentan con jornadas laborales extensas, factores estos que podrían favorecer el sufrir heridas y complicaciones de las mismas.

Con respecto a disponibilidad de recursos económicos para el cuidado de la herida, contar con cuidadores, tener afiliación a la seguridad social y el estrato socio económico en que viven los participantes, no se encontraron datos de otros estudios que permitieran la comparación.

Los hallazgos relacionados con el predominio de heridas con tamaño de seis a diez cms; difiere de los hallazgos de Ribu (6) quien reporta un predominio de ulcera con tamaño menor o igual a un centímetro cúbico.

La ausencia de dolor relacionado con la herida en la mayoría de los participantes difiere de los hallazgos de Da Silva (24), y podría explicarse 
por la presencia de neuropatía, utilización de apósitos de biotecnología avanzada y un mayor tiempo de permanencia en el programa de heridas complejas de SIME E.U.

El alto porcentaje de limitación física relacionada con la herida coincide con lo reportado por Da Silva (24). Así mismo, la presencia de amputación relacionada con la herida fue mayor en este estudio que la reportada por Ribu (5). Este dato podría tener relación con los bajos niveles de escolaridad y con la actividad ocupacional predominante; la que podría haber favorecido la complicación del proceso de cicatrización, llevando a una amputación. No se encontraron resultados de otras investigaciones sobre las fases de cicatrización y presencia de infección.

No se encontraron estudios que informen sobre la puntuación de la CVRS, que relacionaran las distintas dimensiones de la CVRS en personas con heridas, ni que emplearan el "Índice Multicultural de Calidad de Vida", para comparar los resultados con los del presente estudio.

El bajo promedio de CVRS reportado podría estar influenciado por las características socio-demográficas del grupo, tales como el bajo nivel de escolaridad, predominio de trabajos no formales y actividad del hogar, predominio de la no disposición de recursos económicos para el cuidado de la herida. Lo anterior podría sustentarse, mediante lo planteado por Oleson (26), quien considera que la calidad de vida puede estar influenciada por indicadores objetivos (bajas condiciones económicas y de escolaridad) e indicadores subjetivos, que están relacionados con la experiencia de vida de cada uno.

Además de lo anterior, otro aspecto que podría estar relacionado con el bajo promedio de CVRS, es el predominio de limitación física asociada a la herida, dado que según lo planteado por Gonzales (2), la CVRS de las personas puede verse influenciada por la presencia de heridas, en especial si esta implica cambios en la vida diaria, sufrimiento, dolor, limitaciones, dependencia, alteración de la autoestima, temor, angustia y aislamiento, entre otros.

Igualmente los hallazgos del estudio podrían ratificar los referentes teóricos de autores como Naugton (27), quienes consideran que la CVRS 
es la percepción subjetiva, influenciada por el estado de salud actual, de la capacidad para realizar aquellas actividades importantes para el individuo. En ese sentido la presencia de una herida se constituye en un problema físico de salud, la cual es causante de limitación física, y ello dificultaría la realización de actividades que para estas personas son importantes, como la atención del hogar y realización de trabajos que permitan su sustento diario (predominante en el grupo), y esto a su vez los hace percibir una mala CVRS.

La alta puntuación obtenida en la dimensión espiritual se podría apoyar en las afirmaciones de Haynes y Foley $(28,29)$, los que consideran que quienes no han consolidado sus creencias religiosas lo pueden hacer en tiempo de crisis, ya que vuelven a la religión por ayuda, como una fuente de esperanza y fortaleza.

Las puntuaciones altas reportadas en las dimensiones de apoyo socialemocional y funcionamiento interpersonal podrían estar fundamentadas en que la mayoría de los participantes manifestó estar casados o vivir en unión libre, lo que supone contar con una familia. Parece ser que el apoyo social favorece el manejo de la intervención preventiva, terapéutica y rehabilitadora de problemas de salud $(30,31)$.

Otro elemento, ser aspectos concernientes con el entorno cultural y la idiosincrasia de las personas de la región Caribe colombiana que se caracterizan por ser extrovertidas, comunicativas y deliberantes; rara vez solitaria y, en cambio, siempre solidaria, hospitalaria y generosa, en la abundancia o en la pobreza y pródigo para el agasajo (32).

En resumen, se puede concluir que los participantes percibieron su CVRS como mala, resaltando con baja puntuación las dimensiones de plenitud personal, funcionamiento ocupacional, bienestar físico y psicológico.

Las dimensiones de la CVRS que mostraron mayores promedios fueron plenitud espiritual, apoyo social-emocional y funcionamiento interpersonal.

Se evidenció predominio del sexo femenino, ser casados, baja escolaridad, con ocupaciones de tipo informal y en el hogar, no disponer de recursos económicos para el cuidado de la herida compleja, vivir en 
estratos dos y tres, disponer de cuidadores y contar con afiliación a la seguridad social en salud.

Las heridas complejas se caracterizaron por presentar mayor porcentaje en la fase proliferativa de la cicatrización, medir de seis a diez centímetros, no presentar infecciones, no presentar amputación ni dolor relacionado con la herida y presentar limitación física relacionada con la herida.

Se sugiere continuar investigando y publicando sobre el tema dado que existe poca literatura al respecto, lo cual fue una limitación para este estudio

\section{REFERENCIAS}

1. Schwartzmann L. Calidad de vida relacionada con la salud: aspectos conceptuales. Ciencia y Enfermería 2003; 9(2):9-21.

2. Gonzales V. Gerencia del cuidado: Liderazgo de la enfermera en el cuidado de las personas con alteración de los tejidos. Revista Avances de enfermería. 2007; 25(1):122-129.

3. Armstrong D, Lavery L, Wrobel J y Vileikyte L. Quality of life in healing diabetic wounds: does the end justify the means. The Journal of Foot \& Ankle Surgery. 2008; 47(4):278-282.

4. Ragnarson G, Apelqvist J. Health-related quality of life in patients with diabetes mellitus and foot ulcers. Journal of Diabetes and Its Complications. 2000; 14: 235-241.

5. Ribu L, Birkeland K, Hanestad B, Moum T, Rustoen T. A longitudinal study of patients with diabetes and foot ulcers and their health-related quality of life: wound healing and quality of life changes. Journal of Diabetes and Its Complications. 2008; 22: 400407.

6. Ribu L, Rokne B, Moum T, Birkeland K, Rustoen T. Health-related quality of life among patients with diabetes and foot ulcers: associaton with demographic and clinical characteristics. Journal of Diabetes and Its Complications. 2007; 21: 227-236.

7. Ribu L, Rustoen T, Birkeland K, Hanestad B, Paul S, Miaskowski Ch. The prevalence and occurrence of diabetid foot ulcer pain and its impact on health-related quality of life. The Journal of Pain. 2006; 7(4):290-299.

8. Meijer J, Trip J, Jaegers S, Linksoe T, Smits A, Groothoff J, Eisma W. Quality of life in patients with diabetic foot ulcers. Disability and Rehabilitation. 2001; 23(8):336-340.

9. Esteve M, Roca J. Calidad de vida relacionada con la salud: un nuevo parámetro a tener en cuenta.. Med Clin (Barc). 1997; 108: 458-459.

10. De Souza M, De Araújo Z, Marchiori P. Qualidade de vida e saúde: um debate necessário. Ciencia e Saúde Coletiva. 2000; 5(1): 7-18.

11. Botero E, Pico M. Calidad de vida relacionado con la salud en adultos mayores de 60 años: una aproximación teórica. Hacia la Promoción de la Salud. 2007; 12:11-24.

12. Ramirez R. Calidad de vida relacionada con la salud como medida de resultados en salud: revisión sistemática de la literatura. Revista Colombiana de Cardiología. 2007; 14(4): 207-222.

13. Peterson S, Bredow T. Health-Related quality of life. En: FALTAN AUTORES Middle Range Theories. Application to Nursing Research. Lippincott Williams \& Wilkins. New York. 2004. 
14. Mezzich J. The spanish versión of the quality of life index: presentation and validation. $J$ Nerv Ment Dis. 2000; 188(5):301-306.

15. Jatuff D, Zapata-Vega M, Montenegro R, Mezzich J. El índice multicultural de calidad de vida en Argentina: un estudio de validación. Actas Esp. Psiquiatr. 2007; 35(4):253258.

16. Schwartz K, Zapata-Vega M, Mezzich J, Mazzotti G. Validation study of the multicultural quality of life index (MQLI) in a peruvian sample. Rev Bras Psiquiatr. 2006; 28 (1):24-8.

17. Valle R, Zúñiga M, Tuzet C, Martínez C, De la Jara J, Aliaga R, et al. Sintomatología depresiva y calidad de vida en pacientes mujeres con cáncer de mama. An Fac Med Lima, Universidad Nacional Mayor de San Marcos. 2006; 67(4): 327-332.

18. Bulacio J, Vieyra M, Álvarez C, Benatuil B, Mongiello E. Índice de calidad de vida: validación en una muestra argentina. Revista Argentina de Clínica Neuropsiquiátrica. Fundación Argentina de Clínica Neuropsiquiátrica. 2004; 11(4):1-7.

19. Hurtado F, Gómez M, Donat F. Transexualismo: reasignación de sexo y calidad de vida. Sexología Integral 2006; 3(1): 25-34.

20. González R, Calzadilla L, Amador J, Miguez B, Caravia F, Vargas F, et al. Calidad y estilo de vida en estudiantes de medicina y enfermería. Rev. Hosp. Psiquiátrico de la Habana. 2004; 1(1):1.

21. Ministerio De Salud. Resolución 08430 de 1993 por la cual se establecen normas científicas, técnicas y administrativas para la investigación en salud. Bogotá. 1993.

22. Declaración de Helsinki. [Internet]. Disponible en: http://www.isciii.es/htdocs/terapia/ documentos/Declaracion_de_Helsinki.pdf Consultado Julio de 2009.

23. Colombia. Congreso de Colombia. Ley 911 de 2004, octubre 5, por el cual se dictan disposiciones en materia de responsabilidad deontológica para el ejercicio de la profesión de enfermería en Colombia; se establece el régimen disciplinario correspondiente y se dictan otras disposiciones. Bogotá: El Congreso; 2004.

24. Da Silva LL, Trevisan MJ, Do Carmo Cruz M. Qualidade de vida dos portadores de ferida em membros inferiores-úlcera de perna. Revista Ciencia y Enfermería. 2008; 14(1):43-52.

25. Najman M, Levine S. Evaluating the impacto of medical care and technologies on the qualite of life: a review and critique. Social science \& medicine. 1981; 15(1): 107115.

26. Oleson M. Subjectively perceived qualite of live. Image J Nurs Sch. 1990; 22(3): 187-190.

27. Naughton M, Shumaker S, Anderson R, Czajkowski S. Psychological aspects of HealthRelated Quality of Life Measurement: Tests and Scales. Quality of Life and Pharmaco economics in Clinical Trials. 1996; 15:117-131.

28. Haynes F, Watt P. The lived Experience of Healthy Behaviors in people with Debilitating Illness. Holistic Nursing practice. 2008; 22(1):44-53.

29. Foley D. Eleven interpretations of personal suffering. J of Religios and Health.1988; 27:321-328.

30. De La Revilla L. Conceptos e instrumentos de la atención familiar. Barcelona: Ediciones Doyma, SA; 1994. pp. 79-81.

31. Sánchez M. Apoyo social en momentos de crisis. $2^{\mathrm{a}}$ edición. Barcelona: Saludmédica; 2000. pp. 150-153.

32. Martínez L. Dimensiones culturales del hombre costeño. Bogotá: Ediciones Olimpo; 1997. pp. 35-36. 